\title{
A Study on Professional Identity of Non-Native Chinese Teacher
}

\author{
Bo $\mathrm{Yu}$ \\ ${ }^{1}$ International College of Chinese and Culture, Chongqing Normal University, Chongqing 401331, \\ China; \\ ${ }^{2}$ Faculty of Humanities, University of Kelaniya, Kelaniya 11600, Sri Lanka. \\ yubo307@126.com
}

\begin{abstract}
Keywords: professional identity; "mirror image of others"; non-native Chinese teachers; native Chinese teachers.
\end{abstract}

\begin{abstract}
In the field of teaching Chinese as a foreign language, native Chinese teachers, as an important image of "the other", have an impact on the self-image and professional identity of nonnative Chinese teachers. In the non-target language environment, the native Chinese teachers have brought about the discourse of conflict and competition to the non-native Chinese teachers in the aspects of the micro context and the macro discourse through the reference perspective of the "mirror image of others". In order to help build a better professional identity, it is suggested that the non-native Chinese teachers should improve their Chinese skills, enhance their sense of belonging in career and establish positive occupational values, so as to support their career. At the same time, it is necessary to focus on developing the native advantages of non- native Chinese teachers into favorable resources so as to meet the needs of their own career development.
\end{abstract}

\section{Introduction}

The comparative study of native English teachers and non-native English teachers has gradually become a hot spot of foreign research since 1990s (Davies, 1991; Medgyes, 1992), which has a profound influence on the research on foreign language education. In the same way, because of the wide use and popularization of Chinese in the world, the proportion of the studies on non-native Chinese teachers has been increasing in the field of teaching Chinese as a foreign language in the past decade. How the non-native Chinese teachers should recognize their personal identity and professional identity has an important influence on the teachers' professional development and teaching level. Compared with native Chinese teachers, the non-native Chinese teachers face more difficulties and challenges, so that their identity also becomes more complex. In the non-target language environment, there must be some connections and influence between the native language teachers and non-native language teachers. This paper takes the native Chinese teachers as a reference to explore how the non-native Chinese teachers are affected by the native Chinese teachers to build their own professional identity.

\section{Review of Literature}

Identity is the cognition of individuals on which group they belong to. In the field of education, identity is a useful analytical tool for the study on teachers' personal and professional life, as well as the formation of teachers' emotions and practice (Clarke, 2009). As far as its essence is concerned, the identity of foreign language teachers has the characteristics of dynamic change, environmental shaping, interactive association and discourse construction. Xun Yang (2014) holds that the identity of a foreign language teacher should include four dimensions that is, professional identity, major identity, personal identity and situated identity (XUN Yang, SUN Limping Fang, 2014). Among them, professional identity is used to distinguish the external attributes of one group from another group, which pays attention to the problem of how individuals look at themselves, as a way for the individuals to play a professional role in different environments (Coldron, J. \& Smith, R.,1999). 
Braine (1999) believes that there is a general process of self-identification among non-native language teachers, and the shortage or confusion of their identity may lead to a low self-confidence. His views have revealed the importance of understanding the professional identity of non-native language teachers. In terms of the number of people learning Chinese as a foreign language, it has been constantly growing in recent years. By the end of 2016, China has set up 1, 066 examination spots of Chinese Proficiency Test (HSK) in 125 countries and regions all over the world (Confucius Institute Headquarters,2017). The large number of Chinese learners needs sufficient teachers, and it is far from being able to solve the problem to only depend on the domestic output for Chinese teachers, so that the native teachers overseas who don't take Chinese as a mother language will become the main force. As a result, the relevant research on the non- native Chinese teachers should become the focus of the current researchers.

\section{Definition}

At present, in the field of teaching Chinese as a foreign language, little attention has been paid to the education of the non-native Chinese teachers. Generally speaking, the non-native Chinese teachers consist of two groups. one group is the Chinese teachers of ethnic minorities in China who don't take Chinese as the mother tongue, and the other is the native Chinese teachers overseas who don't take Chinese as the mother tongue ((Non-native Chinese Teacher, hereinafter referred to as NNCST). This paper studies the latter. Moreover, in order to investigate the influence process of native Chinese teacher (hereinafter referred to as NCST) on its identity construction, while NCST in particular refers to the Chinese teachers that are sent from China to Confucius Institutes overseas in the context of this paper.

\section{Method and Data Collection}

This paper mainly studies the case of the NNCST from three universities and two secondary schools in Sri Lanka. Sri Lanka is an important country in South Asia. Since the first Confucius Institute was established in 2007, the Chinese language has entered the country's educational system in a short time and established a relatively perfect Chinese communication system (YANG Gang,2016). With the strengthening of the cooperation between China and Sri Lanka, the Chinese language has made considerable progress there in recent years, and the number of non-native Chinese teachers has been increasing. The author once worked in the local Confucius Institute, and had many opportunities to contact with non-native Chinese teachers in Sri Lanka. Thus, there is a realistic foundation to carry out this study. This research adopts the survey method, and data collection is divided into two stages, investigation and interview. In the first stage, a total of thirty people were chosen as respondents, who are all Sri Lankan and speak Sinhalese as their mother tongue. In the second stage of the interview, ten of the thirty respondents were selected, eight are women and two are men. The ten respondents all have experience in studying in China, and they all have experienced the Chinese lessons by NCST and NNCST in the process of learning Chinese. 7 of them have received the training of vocational skills in Chinese.

In the first stage, the questionnaire was conducted by Likert scale, and quantitative data was collected by the SPSS. The scale consists of a set of declarative sentences, and each statement has five answers, corresponding to one to five points respectively. The total score reflects the strength of the attitude of the respondents. In the second stage, personal interviews were carried out to collect qualitative data. The interview was carried out in a semi-structured way. Each interview took about thirty minutes on average, and the process was recorded. And then the relevant content was put in order in the transcription, which was divided into different themes. 


\section{Finding and Analysis}

\subsection{Learning Experience of the Chinese Language.}

In the questionnaire, the respondents were asked to recall the excellent teachers in their experience of learning, and to determine the three major characteristics of these teachers from a series of available items. $63.5 \%$ of the respondents chose Chinese teaching skills, followed by professional Chinese knowledge (52.1\%) and attitude towards students (47.4\%). In addition, about $75.5 \%$ of the respondents said that they gained more knowledge of Chinese from NCST. 68.4\% of the participants said they preferred to be taught by NCST, while $16.3 \%$ said they did not like NCST. Generally speaking, in the process of learning Chinese, the respondents were more affected by native Chinese teachers.

\subsection{Self-Evaluation of Chinese Proficiency.}

$21.2 \%$ of the respondents were satisfied with their own levels of Chinese proficiency, and $57.2 \%$ of the respondents were not satisfied. $85 \%$ of the respondents believed that Chinese proficiency should be listed as one of the important qualifications for Chinese teachers to hold the post. $83.2 \%$ of respondents said that Chinese pronunciation is the most difficult part for them to grasp, especially the intonation of Chinese. So they are not confident in learning pronunciation. Combined with the interview content, the respondents generally had a low self-evaluation on their level of Chinese proficiency, especially when NCST is used as reference.

\subsection{Vocational Skill Training in Chinese Teaching.}

The survey results show that $72.4 \%$ of the respondents who received vocational training courses was mostly related to Chinese proficiency. $62.1 \%$ of them accepted the training from the courses related to Chinese teaching methods. In contrast, knowledge of Chinese literature, Chinese culture and history is not reflected in skills training courses. The result reflects that the knowledge and proficiency of Chinese are the main focus for the respondents to accept the vocational skill training taught in Chinese. Up to $82.8 \%$ still identified the level of Chinese proficiency as the most desirable ability prior to starting their career. In the interview, a number of participants have expressed great expectations for the "standard" Chinese and regarded the proficiency of Chinese similar to that of their "mother tongue" as the goal of their professional training.

\subsection{Position and Belief of the Chinese Language.}

A majority of the participants $(71.1 \%)$ agreed or strongly agreed that Chinese is the most important foreign language in Sri Lanka (except for English). With the development of relations between China and Sri Lanka, there have been an increasing number of Sri Lankans learning Chinese, and the career prospects of local Chinese teachers are highly rated. Many young people in Sri Lanka regard Chinese as such a popular language that they are very eager to learn it. Learning Chinese is of great significance to their future job hunting, business dealings, and the search for greater space for their own development, which is an important motivation for the learners to learn Chinese. Moreover, the professional Chinese learners are able to have some better employment channels. Women are more inclined to serve as teachers. The motivation of learning Chinese influences their cognition of the status of Chinese, and also affects the construction of their professional identity.

\subsection{Professional Image of NNCST.}

When asked about what qualities their desirable Chinese teachers should have, more than half of the respondents $(63.8 \%)$ chose the Chinese proficiency, which reflects that the majority of respondents believe that Chinese proficiency is the most important criterion for assessing the professional image. For lots of respondents, the Chinese proficiency mainly refers to standard pronunciation and fluent expressions. What's more, the idea professional image of Chinese teachers must be able to provide the standard pronunciation and intonation for the students. About half of the respondents $(52.4 \%)$ thought they were the ideal professional image of Chinese teachers.

The survey findings have further shown that $64.4 \%$ of the respondents believed that they could become excellent Chinese teachers. However, when asked to compare with NCST, less than half $(37.3 \%)$ of the respondents believed that they could be the same as NCST. As a matter of fact, $45.9 \%$ of the respondents held a neutral attitude towards this question. In the interviews, it was quite difficult 
for many of the respondents to make a comprehensive judgment on the teaching of NCST, because the two kinds of teachers have had different advantages.

The existence of NCST may affect the self-image and self-perception of NNCST, which will make NNCST feel stressed in the work, so that they may be at a disadvantage in Chinese teaching and have a weak sense of belonging to their career.

\subsection{Recognition of NCST and NNCST's Merits and Drawbacks.}

\subsubsection{NCST.}

In terms of the merits of NCST, $89.4 \%$ of the respondents agreed that NCST is good at teaching the knowledge about Chinese culture. $90.4 \%$ of the respondents believed that NCST does well in pronunciation. Another $88.1 \%$ believed that NCST is good at teaching oral skills. In addition, $65.5 \%$ of the respondents agreed or strongly agreed that NCST is good at teaching listening skills. These results have shown that most of the respondents believe that the instructions with regard to pronunciation, oral and Chinese culture teaching are the main advantages of NCST. In the same way, when most of the respondents talked about NCST's ability to teach related pronunciation skills, they strongly expressed their preference for "native speakers". In terms of NCST's drawbacks, the respondents believed that the NCSTs are lacking the knowledge of the local context, and that they are unable to speak the first language of the students, and they are short of the experience of learning Chinese as a foreign language as well as the solid grammar knowledge.

\subsubsection{NNCST.}

The results of the survey showed that $73 \%$ of the respondents believed that NNCSTs are good at teaching grammar. $80.9 \%$ of the respondents believed that they are good at teaching students the skills for an examination, $80.7 \%$ thought they are good at teaching translation skills, and $78.2 \%$ thought they are good at teaching learning strategies. By contrast, most of the respondents $(70.4 \%)$ did not agree that the NNCSTs are good at teaching writing and vocabulary. In the interview, a large number of the respondents mentioned that, as NNCSTs, they are familiar with the local educational system and culture, and thus they are able to adopt some effective teaching skills to enable their students to deal with the problems in grammar and then to pass the exam. NNCSTs are able to communicate with students in their mother tongue and to provide certain guidance and advice for students with learning difficulties. As former Chinese learners, NNCSTs understand the difficulties of students in learning Chinese, so that they are able to provide useful learning strategies for the students.

As far as NNCST's drawbacks are concerned, the results of the survey showed that $55.5 \%$ of the respondents agreed or strongly agreed that the NCCSTs are not good at teaching language skills. Most of the respondents $(72.5 \%)$ thought that they are not good at pronunciation. In addition, more than half of the respondents $(65.4 \%)$ thought they are not good at teaching the knowledge regarding Chinese culture. These results indicate that teaching methods and pronunciation are the main weaknesses of NNCST.

\subsection{Evaluation the Social Values and Status of NCST and NNCST.}

$65.2 \%$ of the respondents believed that the government should adopt an access system for NNCST. That is to say, there should be a qualification examination for Chinese teachers or some rigid rules on the Chinese proficiency of the teachers (HSK). Most of the respondents (92.5\%) agreed or strongly agreed that NCST should also obtain a qualification certification, and it would be best to have an official Chinese teacher qualification approved by the Chinese government.

As far as social attitudes towards NCST and NCEST are concerned, up to $71.6 \%$ of them believed that Chinese learners in Sri Lanka prefer to be taught by NCST. As the policies in the communications between China and Sri Lanka are becoming favorable, cultural and educational exchanges are becoming increasingly close, and the number of NCSTs has gradually increased, resulting in some competitive pressure to the NNCSTs to a certain extent. In the interview, the respondents mentioned that Chinese learners and their parents thought that NCST is better than NNCST. In the absence of their qualifications or experience, NCST has a natural advantage. At least in the initial stage of Chinese learning, they can be guided by the "authentic" intonation for learning the pronunciation. As many as $92.8 \%$ of the respondents believed that in Sri Lanka, NCSTs have a 
higher salary than NNCSTs and they are not very satisfied with the current salary standard, and there is a gap between their salaries and those of the teachers in other foreign languages. $75.2 \%$ of the respondents believed that NNCSTs have local advantages, and they are more likely to find part-time jobs than NCST (such as interpreter and tour guide). These results indicate that NNCST is more competitive in the job market, but their social prestige and status are not directly proportional to each other, and their advantages are not prominent. More than half of the respondents (51.2\%), considering that they are not favored by local learners, admit that they are worried about the future of Chinese teachers because NNCSTs don't have prominent competitiveness in the job market.

In addition, NNCST is likely to suffer an imbalance in the "power" relationship with NCST during the work, leading to the anxiety of the former in losing the "power" in development and the depreciation on their serviceability as a Chinese teacher. The respondents said that they have often had conflicts with NCSTs in terms of educational teaching and curriculum goals. The existence of NCST will have an impact on their own confidence in Chinese language proficiency, resulting in their concerns about the negative judgments by their colleagues and students due to the language proficiency and "mother tongue status".

\section{Conclusion}

Identity is the self consciousness of an individual, which is formed from the reflection of "the other". Without the reflection of "the other", we will be unable to form self consciousness (DENG Changjie XU Xingchun,2016). Such a mirror effect is an important way to construct identity. NCST, as a mirror image of "the other", has set up an ideal image of a professional Chinese teacher for NNCST. The results show that the emergence of NCST helps NNCST to define and understand their strengths and weaknesses in teaching, through the reference of "the mirror image of the other", they have already realized that they have advantages in grammar teaching, teaching skills for examinations and guidance on learning strategies. At the same time, they regard the Chinese proficiency, especially related pronunciation skills, as the main weakness of NNCST. As a reference, they think that NCST has "authentic" use of vocabulary, pronunciation and intonation. On the other hand, for most of the respondents, standard pronunciation is one of the most important features in the professional abilities of the best teachers, as well as the core element of the professional image of an ideal Chinese teacher. Professional identity is associated with teachers' self -concept and self-image, which plays an important role in the teachers' teaching methods, professional development and their attitudes towards the changes in education (ZHANG Min, 2006). NNCST is faced with the discourse of competition in the occupational market brought by NCST. On the one hand, as a qualified Chinese teacher, both NNCST and NCST need to be certified and obtain professional training, which has made the Chinese teachers themselves have a sense of competition.As language educators, NCST has higher expectations from the school organizations and social groups. NNCST is at a disadvantage in the job market. What's more, it is difficult for NNCST to build its own professional identity in the local context. The respondents hold that the belief of becoming a Chinese teaching professional is also challenged by the general acceptance of the society, and the social groups have laid excessive stress on the native language background of Chinese teachers. For NNCST, its own local advantages have not been attached importance to by the organizations and society.

\section{References}

[1]. Braine, G. (Ed.). (1999a). Non-native educators in English language teaching. Mahwah, NJ: Lawrence Erlbaum.

[2]. Clarke, M. (2009). The ethico-politics of teacher identity. Educational Philosophy and Theory. 41(2), p:185-200.

[3]. Coldron, J. \& Smith, R. (1999). Active location in teachers, construction of their professional identities. Journal of Curriculum Studies.31(6), p:711-726. 
[4]. Confucius Institute Headquarters (2017). Confucius Institute Annual Development Report 2016, p: 6

[5]. Deng Changjie,XU Xingchun(2016).An Analysis of the Feasibility of Constructing Preschool Teachers Identity Based on"Others Mirror Image"and"Self-identity”, Education and Teaching research.30(7), p: 46-50.

[6]. Sun Yang,SUN Li,PENG Fang(2014).Formulation and Validation of a Teacher Identity Inventory for EFL Teachers,Shandong Foreign Language Teaching Journal. 162(5), p: 61-67.

[7]. Yang Gang (2016). Spread of the Chinese Language in Sri Lanka in ancient times, Journal of Yunnan Normal University (Teaching and Research on Chinese as a Foreign Language Edition).14(5), p: 86

[8]. Zhang Min(2006) .On Foreign Teachers' Professional Identity and their Professional Development, Comparative Education Review.189(2),p:77-81 